\section{College of Anaesthetists}

SIR,-The letter signed by a number of leading anaesthetists (4 March, p 574) comments inter alia on the passage in my editorial in Anaesthesia suggesting that those favouring the proposal for an independent College of Anaesthetists "will wish to ensure that those whom they elect to the Board of Faculty . will also reflect their views and facilitate a smooth transition to independence" by stating that "it would seem that this is encouragement to elect to the Board of Faculty of Anaesthetists those willing to disregard their declaration of loyalty to that body which, in fact, they clearly plan to disrupt."

The letter from the President and Deans states: "There is nothing ignoble in such sentiments [that is, the desire for the development of an independent College of Anaesthetists], nor do we regard those who hold them as being in any way disloyal to the College [of Surgeons of England] . ..."

I made it quite clear in my editorial that neither I nor others who seek an independent College of Anaesthetists seek to disrupt the Faculty. We merely hope to see gradual and amicable evolution towards an independent College of Anaesthetists. There is nothing disloyal in this sentiment.

T B BoultoN Editor, Anaesthesia Association of Anaesthetists of
Great Britain and Ireland,
London WC1

\section{Fall in admission rate of old people to} psychiatric wards

SIR,-I was interested to read the paper by $\mathrm{Dr}$ K Shulman and Professor Tom Arie (21 January, p 156) and subsequent correspondence (4 February, p 299). From the data Dr Shulman and Professor Arie present I think their conclusion concerning changes in firstadmission rates is unwarranted, for three reasons.

(1) They describe the dramatic change in definition of "first admission" which occurred in 1970 but suggest that this change was not the sole reason for the very large reduction in first-admission rates recorded in $1970 \mathrm{com}-$ pared with 1969. Basic scientific method indicates that a substantial (or even any) change in definition of the events recorded prevent conclusions being drawn across the times of change. Without collecting 1970 firstadmission data using both definitions there is no way of knowing whether the change in recording first admissions in 1970 produced any, some, or all of the dramatic drop in rates.

(2) They say, in favour of their inference that first admissions dropped only from 1970, that if this drop (according to the 1970 definition) was actually happening from 1965 onwards it would have been reflected by the total admission rates also dropping from 1965. This is not necessarily so. It would be perfectly possible, for example, to maintain (or increase) total admission rates by a policy of planning regular readmissions for short periods of time

(3) Looking at 1970-4 data it appears that there is a falling trend in first admissions of elderly people for all diagnoses (their fig 1) Let us assume though, for the sake of argument, that the 1970 drop in first admissions is wholly artefactual and adjust the rates for 1970 to 1974 accordingly by adding to each one the difference between the 1969 and 1970 figures. (This can be done visually simply by sliding the 1970-4 sections of the graphs upwards till 1970 is level with 1969). It can now be seen that the first admissions between 1970 and 1974 lie largely within the range of first admissions recorded between 1965 and 1969 . Using this assumption (perfectly reasonable in view of the definition change), it is difficult to assert that first-admission rates are showing a steady downward trend from 1970 to 1974 in relation to $1965-9$ rates. For first-admission rates of people with diagnoses of dementia the drop shown between 1970 and 1974 (their fig 2) may well be, similarly, within the range of rates between 1965 and 1969. Dr Shulman and Professor Arie do not give us firstadmission rates for this group of people for the years 1965-9, though total numbers are given in their table II. It would be helpful, in considering the degree to which first admissions for dementia may have dropped, to have the table II data transformed into rates, thus providing a more direct comparison with table I and fig 1. (This would not, of course, avoid the definition change problem.)

There may indeed be a falling rate in first admissions to psychiatric units, and the authors' reasons for it may well all be true. It is not possible, however, to deduce these conclusions and reasons from the data presented in the paper. It seems to me that the most direct (though not simple) way of finding out what is happening to elderly people categorised as demented and their families is to look at them in "real life," not to hypothesise from inevitably crude national statistics.

JUDITH JENKINS

Health Care Evaluation

Research Team,
Winchester, Hants

\section{Inhaling habits of pipe smokers}

SIR,-The question to which Dr J A McM Turner and his colleagues sought an answer in the experiment reported recently in your columns (26 November, p 1387)-namely, "Do inhaling cigarette smokers continue to inhale when they switch to cigars?"-is an important one. It is also important, therefore, to be sure that their conclusion that "excigarette smokers do not seem to lose their habit of inhaling when they change to cigars" is justified.

Ideally, to answer the question one would want to compare persons who smoke cigarettes and nothing else and then switch to cigars and nothing else with persons who have always smoked cigars and nothing else and continue to do so. Dr Turner and his colleagues did not do this. Their "ex-cigarette smokers" group consisted of three former pure cigarette smokers who had switched to cigars only and two former mixed pipe and cigarette smokers, one of whom took up cigars after giving up cigarettes. Their "primary pipe and cigar smokers" on the other hand consisted of five people who not only had always smoked pipe tobacco but who also were, at the time of the study, predominantly pipe smokers Counting large cigars as weighing $6.5 \mathrm{~g}$, medium as $4.5 \mathrm{~g}$, and small as $2.25 \mathrm{~g}$, none of the five smokers took more than $39 \%$ of his tobacco from cigars and most took much less. In view of this and the fact that the level of self-reported inhaling of pipe smokers is known to be markedly less than that of cigar smokers, ${ }^{1}$ it was only to be expected, regardless of past smoking history, that the level of inhaling in this group would be less than that in the "ex-cigarette smokers," only two of whom had ever smoked pipes.

Apart from this we are puzzled by the high plasma nicotine levels before smoking in the ex-cigarette smokers considering that three of them had abstained from smoking for at least two days before the study and the remaining two had abstained for $6 \mathrm{~h}$ or more. The average resting value in this group was almost four times that in the other group. Moreover, the highest value observed $(207.4 \mathrm{nmol} / 1$ $(33.6 \mathrm{ng} \mathrm{ml}))$, apart from being highly significantly outside the range of the values found in the primary pipe and cigar smokers $(\mathbf{P}<0.001)$, seems inconsistent with the reported half life for nicotine, which is under $30 \mathrm{~min}, 2$ and the very much lower resting values of non-smokers. ${ }^{3}$

London SW 19

F J C RoE

Tobacco Research Council,

P N LEE

London SW1

Statistics of Smoking in the United Kingdom, ed P N Lee, Tobacco Research Council Research Paper No 1, 7th edn. London, TRC, 1976

2 Isaac, P F, and Rand, M J, Nature, 1972, 236, 308.

Russell, M A H, and Feyerabend, C, Lancet, 1975, 1 ,

***We sent a copy of this letter to Dr Turner and his colleagues, whose reply is printed below-ED, BMF.

SIR,-We were interested to read the comments of Dr Roe and Mr Lee.

We, of course, agree that ideally it would be better to study "pure" ex-cigarette smokers smoking cigars, and "pure" primary cigar smokers, but, as Dr Roe and Mr Lee appreciate, the majority of smokers have at some time in their life been "mixed" smokers. We therefore feel that our observations regarding ex-cigarette smokers who switch to cigars are valid.

We also expected the level of inhalation in the primary pipe/cigar smoker group would be lower than in the ex-cigarette group, but we did not expect to find that there would be no significant inhalation in this group while smoking cigars. The maximum increase of carboxyhaemoglobin during smoking in this group was $0.8 \%$. (Mean pre-smoking carboxyhaemoglobin $0.8 \%$; mean smoking carboxyhaemoglobin $1.0 \%$ ) These increases are similar to those in non-smokers reported by Russell et $a l^{1}$ being due to passive smoking.

The high mean pre-smoking nicotine concentration in the ex-cigarette group was due to the two subjects who had only abstained for $6 \mathrm{~h}$ before the study. The plasma nicotine concentrations in the other three ex-cigarette smokers were similar to those of the primary pipe and cigar smokers. The half life of plasma nicotine has been shown by Armitage et al to range from 24 to $84 \mathrm{~min}$ with a mean of 40 min. Our data suggest that the plasma nicotine half life is in excess of $60 \mathrm{~min}$; the mean percentage fall in plasma nicotine concentration one hour after smoking was $32 \%$.

\section{J A MCM TURNER R W SilletT $\mathrm{M}$ W MCNICOL}

Middlesex Hospital, London W1

Russell, M A H, Cole, P V, and Brown, E, Lancet $1973,1,576$ Armitage, A K, et al, British Medical fournal, 1975 ,
4, 313 . 